\title{
PROSPECTIVE ASSOCIATIONS BETWEEN METH/AMPHETAMINE (SPEED) AND MDMA (ECSTASY) USE AND DEPRESSIVE SYMPTOMS IN SECONDARY SCHOOL STUDENTS
}

Frédéric N. Brière, Ph.D. ${ }^{1,4}$, Jean-Sébastien Fallu, Ph.D. ${ }^{2,3,4}$, Michel Janosz, Ph.D. ${ }^{2,4}$, Linda S.

$$
\text { Pagani, Ph.D. }{ }^{2,4,5}
$$

${ }^{1}$ Département de Psychologie, Université de Montréal.

${ }^{2}$ École de Psychoéducation, Université de Montréal

${ }^{3}$ Institut de Recherche en Santé Publique de l'Université de Montréal (IRSPUM)

${ }^{4}$ School Environment Research Group (SERG), Université de Montréal, Canada

${ }^{5}$ Centre de Recherche de l'Hôpital Sainte-Justine, Université de Montréal, Canada

Correspondence: Frédéric N. Brière, Département de psychologie, Université de Montréal, C.P. 6128, succursale Centre-ville, Montréal, Québec, Canada, H3C 3J7.Telephone: 514-343-6111, extension 2572. (e-mail: frederic.nault-briere@umontreal.ca, permission granted to publish this e-mail address).

Keywords: MDMA, methamphetamine, depression, adolescence, longitudinal Word count: 3234 


\begin{abstract}
Background. Research has raised significant concern regarding the affective consequences of synthetic drug use. However, little evidence from well-controlled longitudinal studies exists on these consequences. The aim of this study is to determine whether use of meth/amphetamine (speed) and $\pm 3,4$-Methylenedioxymeth-amphetamine (MDMA, ecstasy) is independently predictive of subsequent depressive symptoms in adolescents.
\end{abstract}

Methods. A sample of 3880 adolescents from secondary schools in disadvantaged areas of Quebec, Canada, were followed over time (2003-2008). Logistic regression was used to test the association between meth/amphetamine and MDMA use in grade 10 (ages 15-16) and elevated depressive symptoms on an abridged CES-D scale in grade 11, controlling for pre-existing individual and contextual characteristics.

Results. After adjustment, both MDMA use (odds ratio, 1.7; 95\% confidence interval, 1.1-2.6) and meth/amphetamine use (odds ratio, 1.6; 95\% confidence interval, 1.1-2.3) in grade 10 significantly increased the odds of elevated depressive symptoms in grade 11 . These relationships did not vary by gender or pre-existing depressive symptoms. Increased risk was particularly observed in concurrent usage (odds ratio, 1.9; 95\% confidence interval, 1.2-2.9).

Conclusions. Adolescent use of meth/amphetamine and MDMA (particularly concurrent use) is independently associated with subsequent depressive symptoms. Further inquiry must determine whether these associations reflect drug-induced neurotoxicity and whether adolescence is a period of increased vulnerability to the hazards of synthetic drug exposure. 


\section{PROSPECTIVE ASSOCIATIONS BETWEEN METH/AMPHETAMINE (SPEED) AND MDMA (ECSTASY) USE AND DEPRESSIVE SYMPTOMS IN SECONDARY SCHOOL STUDENTS}

\section{INTRODUCTION}

Meth/amphetamine (speed) - i.e. amphetamine or the more prevalent and potent methamphetamine - and $\pm 3,4$-Methylenedioxymethamphetamine (MDMA, ecstasy) are ranked among the most popular illicit drugs consumed by youth in North America and worldwide. Although the use of these two types of drugs is particularly widespread among young club/rave goers in the dance scene, ${ }^{1}$ it has also become commonplace in the general population. ${ }^{2}$ Trends over the past 20 years show increased initiation and regular use among adolescents. ${ }^{3}$ The concurrent use of meth/amphetamine and MDMA is typical among recreational users and often occurs in the same drug-taking episode, either intentionally or not (through tablet impurity).,

Over the past two decades, clinicians and researchers have become increasingly concerned about the putative consequences of meth/amphetamine and MDMA use. Animal models suggest neurotoxicity in response to large doses of MDMA and meth/amphetamine. ${ }^{5}$ In humans, research suggests potential neurological and functional impairments, notably with regards to the development of depressive symptomatology. ${ }^{6-9}$ Neurotoxicity from the two substances on 5-HT neurotransmission could be involved, given that low 5-HT functioning is a trademark biological marker of clinical depression. ${ }^{6}$ The associated depressive outcomes of synthetic drug use in adolescents are particularly disconcerting. First, the brain undergoes a period of extensive neuronal maturation during adolescence, which opens a window of vulnerability to the neurotoxic effects of drug use, even at low doses and frequencies. ${ }^{10}$ Second, depressive 
symptoms in adolescence can potentially disrupt normative development and foster delays in social, emotional, and/or cognitive development. ${ }^{11}$

Nonetheless, relatively little research on the relation between MDMA and meth/amphetamine use and depressive symptoms in adolescents has been published. ${ }^{12-14}$ Existing research has been mostly cross-sectional, although a few recent longitudinal studies provide evidence in support of a relation. ${ }^{12,13,15}$ Confounding factors have been identified as important limitation of most previous studies, in particular pre-existing characteristics and more general drug use. ${ }^{7,16}$ For example, synthetic drug users may have elevated depressive symptoms that precede rather than follow their drug use. Meth/amphetamine and MDMA use have also typically been considered separately, despite the frequent concurrent use and putative additive or interactive noxious effects of these drugs.

In this study, a population-based, longitudinal sample of typically developing adolescents was used to examine the prospective relationship between meth/amphetamine use and MDMA use in tenth grade and subsequent elevated depressive symptoms in eleventh grade. The separate and concurrent consumption of these drugs were considered. In order to secure unbiased estimates, our analytic strategy considers pre-existing individual and contextual factors, including antecedent depressive symptoms and the concurrent use of other substances. 


\section{METHODS}

\section{Participants}

Participants are from the New Approaches New Solutions (NANS) longitudinal data set (20032008). A stratified random sampling strategy was designed to select schools representative of all schools in disadvantaged areas of Quebec in terms of geographical location and size. The initial sample for this study comprises 6126 students from 57 low SES French-language secondary schools across the province of Quebec (Canada). Most participants $(n=5055)$ were followed from grades 7 to 11 which correspond to the first and last year of secondary school in Quebec. A subset $(\mathrm{n}=1071)$ was followed from eighth grade. Consent was obtained for $77 \%$ of the eligible participants. The NANS sample is mostly Caucasian (91.3\%) and approximately represented by gender (54.4\% females). For the present study, the sample included all students aged 15 or 16 with available data in grade 10 , as well as available data at baseline, in either grade 7 or 8 . $(\mathrm{n}=3880)$. Follow-up data in grade 11 from these participants were also used as part of study design. A total of 2161 participants (55.7\%) were available at follow-up in grade 11 . Missing data on background variables collected at grades $7-8$ ranged from $0 \%$ to $22.5 \%$. Written informed consent was obtained with signatures from all parents and participants. All self-reported questionnaires were administered in class by trained and supervised experimenters. All data collection procedures obtained IRB approval by the University of Montreal.

The fact that a proportion of the participants who were part of the initial NANS sample at the beginning of secondary school were excluded based on data non-availability in grade 10 has implications for sample representativity. The reasons for data non-availability in grade 10 are largely similar to the reasons related to sample attrition from grade 10 to 11 . The data analysis section details these reasons and also shows a comparison between study dropouts and 
participants who remained in the sample. As will be seen, study dropouts were globally at higher risk than individuals who remained in the study and for this reason, the final sample should be considered of overall lower risk than the initial NANS population at the beginning of secondary school.

\section{Measurement of MDMA use and meth/amphetamine use}

MDMA use and meth/amphetamine use were assessed in grade 10 using the following items: “Have you ever used ecstasy (MDMA)?" and "Have you ever used speed (amphetamines)?". The response scale for these items was never (0); yes, in my lifetime (1); yes, in the past year (2); and (3) yes, in the past month. Past-year use was obtained by coding the latter two categories as 1 (= yes), and by coding the first two categories as $0(=$ no $)$. Both estimates were also combined to create a four-category variable, in the form of three dummy variables representing MDMA use alone, meth/amphetamine use alone, and use of both meth/amphetamine and MDMA.

\section{Measurement of outcome variable: elevated depressive symptoms}

Depressive symptoms were measured in grade 11 using an abridged version of the Center for Epidemiologic Studies-Depression (CES-D) questionnaire. ${ }^{17}$ The 16-item abridged scale assesses how individuals felt or behaved in the past week $($ alpha $=.89)$. Each item is answered on a 4point scale, ranging from "rarely or none" to "most or all". The scale has been validated for use with adolescents. ${ }^{18}$ To capture symptom severity, depressive symptoms were divided into two categories of "elevated" and "low" symptoms using a total score cut off at 16 or beyond. This cut-off approximately corresponds to $1-\mathrm{SD}$ above the mean as well as the $85^{\text {th }}$ percentile of depressive symptoms in grade 11. This yielded a proportion of participants with "elevated symptoms" which corresponds to depression prevalence estimates observed in adolescents. ${ }^{19}$ 


\section{Measurement of potential confounders}

\section{Concurrent Use of Other Substances.}

Students provided an assessment of concurrent general substance use with MDMA and meth/amphetamine use in grade 10. Frequency of alcohol use and frequency of marijuana use in the past year were assessed using a 7-point scale ranging from "never" to "more than 60 times". The use of hard drugs ("heroin, morphine, opium, crack, etc") in the past year and cigarette use in the past 30 days were assessed as dichotomous measures (no use; any use).

Pre-existing Individual and Contextual Characteristics.

Individual, family, and peer characteristics of participants were considered as controls prior to MDMA use and meth/amphetamine use. When possible, mean scores in grades 7-8 were used to obtain more stable characteristics and reduce missing data (available score was used when one data point was missing). Pre-existing depressive symptoms were measured with the same abridged CES-D scale (alpha $=.92)$ as the outcome measure. Affective engagement to school was measured using 6-item scale rated on a 7-point Likert scale $($ alpha $=.83) .{ }^{20}$ School anxiety was measured using a 6-item scale (alpha $=.82)$, mostly composed of items from Spence's anxiety questionnaire. ${ }^{21}$ Participants rated statements on a 4-point scale. Academic achievement was measured as a mean score of self-reported grades in Language arts (French) and mathematics. Grade retention was dichotomously coded as participants who were not at an age-appropriate level (i.e. participants who were 1 year behind or in a special class) in grade 7 or $8(=1)$ as opposed to participants who were $(=0)$. Delinquency was measured using a 16-item scale investigating delinquent behaviors in the past year $($ alpha $=.94)$, rated on a 4-point scale. ${ }^{22}$ General intelligence was measured using the Standard Raven Progressive Matrices for 
adolescents at first data collection for each participant. ${ }^{23}$ The conscientiousness subscale of the Big Five Inventory ${ }^{24}($ alpha $=.75)$ was used as a proxy reflecting planning and socially appropriate inhibitory control aspects of executive functioning. ${ }^{25}$ Participants rated 9 items on a 4-point scale. The scale was not available in grades $7-8$ and was rather considered as a stable individual characteristic in grade 10.

Contextual controls were variables related to the family and peers of participants. Family variables included conflict with parents (3-item scale, alpha $=.79$ ), positive communication with parents (6-item scale, alpha $=.88)$, and parental rules $(7$-item scale, alpha $=.71) .{ }^{22}$ Information was also collected at first data collection for each participant about maternal education $(0=$ no high school degree; 1 = high school or higher), intact/nonintact household $(0=$ parents together; $1=$

parents separated), and parental occupational prestige (mean of mother and father score). ${ }^{26}$ Three peer controls were included: (1) Drug use by closest friends $(1=$ yes; $0=$ no); (2) Social isolation (5-item on a 4-point Likert scale, alpha =.86); and (3) Peer social support (4-item on a 5-point Likert scale, alpha $=.86) .{ }^{22}$

\section{Data analysis}

Logistic regression, estimated with maximum likelihood (ML), was used to model the unique contribution of MDMA and meth/amphetamine use in grade 10 in predicting subsequent elevated depressive symptoms (CES-D score of 16 or above) in grade 11. First, the separate contribution of MDMA use (Model 1) and meth/amphetamine use (Model 2) in predicting elevated depressive symptoms were tested. Second, MDMA and meth/amphetamine use were investigated together (Model 3) by creating three mutually exclusive predictors: MDMA use alone, meth/amphetamine use alone, and concurrent use (use of both substances, whether in the same drug-taking episode or 
not). Model 3 was selected over a model assessing the relative prediction of MDMA and meth use because the two variables were highly correlated and their simultaneous inclusion as independent predictors yielded unstable estimates with large standard errors. Model 3 resolved this issue and also offered the advantage of directly assessing the risk associated with this concurrent use of both substances for subsequent depressive symptoms. Unadjusted and adjusted versions each model were fitted. Adjusted versions included controls for (1) pre-existing individual and contextual characteristics at baseline (grades 7-8) and (2) concurrent use of other substances in grade 10 (frequency of alcohol and cannabis use, cigarette use, and hard drugs use). Adjusted models were estimated adjusting for the within-school variance tied to the nested design. Information on the link between control variables and the outcomes can be found in Appendix 1 (Web Only File).

Attrition in grade 11 was associated with MDMA use $\left(\chi_{1}^{2}=27.9, p<.001\right)$ and meth/amphetamine use $\left(\chi_{1}^{2}=9.7, p=.002\right)$ in grade 10 , as well as depressive symptoms in grades $7-8\left(\mathrm{t}_{3878}=2.39, \mathrm{p}=.02\right)$, suggesting a potential attrition bias. Further information can be found in Appendix 2 (Web Only File). Multiple imputation was used since it has been shown to reduce attrition bias, especially when the outcome variable is available at multiple time points. ${ }^{27}$ The imputation was conducted with NORM. ${ }^{28}$ All study variables, including interaction terms, were included in the imputation model. Twenty different datasets were imputed and results are averages of parameters in models fitted on all datasets using Mplus version 5.1. ${ }^{29}$ For validation purposes, models 1 to 3 were replicated with continuous CES-D scores and both dichotomous and continuous versions were also fitted on the non imputed dataset. Results were largely similar in terms of direction and significance in all validation models. 


\section{RESULTS}

\section{Descriptives}

Prevalence of MDMA use and meth/amphetamine use in grade 10 and elevated depressive symptoms in grade 11 are reported in Table 1. Meth/amphetamine use was more prevalent than MDMA use. Use of both meth/amphetamine and MDMA (6.7\%) was more prevalent than meth/amphetamine use alone (4.9\%) and MDMA use alone (1.3\%). There were no gender differences in the prevalence of meth/amphetamine use and MDMA use in grade 10, as well as elevated depressive symptoms in grade 11 (CES-D score of 16 or above).

Table 1. Prevalence of MDMA and Meth/amphetamine Use in Grade 10 and Elevated Depressive Symptoms in Grade 11

\begin{tabular}{|c|c|c|c|}
\hline & \multicolumn{3}{|c|}{$\mathrm{n}(\%)^{\mathrm{a}}$} \\
\hline & Females & Males & Total \\
\hline MDMA use & $152(7.2)$ & $158(8.9)$ & $310(8.0)$ \\
\hline Meth/amphetamine use & $236(11.2)$ & $215(12.1)$ & $451(11.6)$ \\
\hline Elevated Depressive Symptoms ${ }^{b}$ & $326(15.5)$ & $258(14.6)$ & $584(15.1)$ \\
\hline
\end{tabular}




\section{Prediction of depressive symptoms by MDMA use and meth/amphetamine use}

Table 2 reports the association between meth/amphetamine use and MDMA use in grade 10 and subsequent elevated depressive symptoms (CES-D score of 16 or above) in grade 11. Models 1 and 2 show that both MDMA use and meth/amphetamine use significantly increased the odds of having elevated depressive symptoms the following year. These relationships remained significant after adjusting for a series of pre-existing individual and contextual characteristics, including depressive symptoms at baseline, as well as the concurrent use of other substances. After adjustment, MDMA users and meth/amphetamine users were respectively 1.7 times and 1.6 times more likely than non-users to report elevated depressive symptoms the following year. Relative to other variables, these associations made the largest contributions toward predicting depressive symptoms after baseline school anxiety and depressive symptoms (though within the range of other significant controls). 
Table 2. Association Between Meth/amphetamine and MDMA use in Grade 10 and Elevated Depressive Symptoms in Grade 11

\begin{tabular}{cc} 
Elevated Depressive Symptoms & \\
& \\
Unadjusted & OR $(95 \%$ CI $)$ \\
\hline & Adjusted $^{\mathrm{b}}$
\end{tabular}

Model 1

MDMA use

$2.4(1.6-3.5)^{\mathrm{c}}$

$1.7(1.1-2.6)^{\mathrm{c}}$

Model 2

Meth/amphetamine use

$2.1(1.5-2.9)^{\mathrm{c}}$

$1.6(1.1-2.3)^{\mathrm{c}}$

Model 3

Meth/amphetamine use only

$1.7(1.1-2.7)^{\mathrm{c}}$

$1.4(0.8-2.4)$

MDMA use only

$2.1(0.9-5.0)$

$1.5(0.6-4.1)$

Concurrent use

$2.5(1.6-3.8)^{\mathrm{c}}$

$1.9(1.2-2.9)^{\mathrm{c}}$

\footnotetext{
Abbreviations: OR, Odds Ratio; CI, Confidence Interval.

${ }^{\text {a }}$ Center for Epidemiologic Studies-Depression (CES-D) score of 16 or above.

${ }^{\mathrm{b}}$ Adjusted for the use of other substances in grade 10 (frequency of alcohol Use, frequency of marijuana use, hard drugs use, cigarette use), conscientiousness in grade 10, and pre-existing individual and contextual characteristics in grades 7-8 (depressive symptoms, affective engagement to school, school anxiety, academic achievement, grade retention, delinquency, intelligence, conflict with parents, positive communication with parents, parental rules, maternal education, intact/non intact household, parental occupational prestige, drug use by best friends, peer social support, social isolation).

${ }^{\mathrm{c}}$ Significant findings.
} 
Model 3 was aimed at better circumscribing the unique and combined contributions of meth/amphetamine use and MDMA use in predicting the outcome. Before adjustment, both concurrent users and meth/amphetamine only users were significantly more likely than non-users of being in the upper end of depressive scores. However, only combined use of MDMA and meth/amphetamine remained significantly associated with subsequent elevated depressive symptoms after adjusting for confounders. After adjustment, combined users were 1.9 times more likely than non-users to report elevated depressive symptoms the following year. Non significance of MDMA and meth/amphetamine use only should be considered with caution, given the low prevalence within these categories (below 5\%).

Because somatic symptoms could reflect transient effects of concurrent drug use in grade 11 rather than long-term depressive symptoms per se, ${ }^{16}$ the above models were replicated using a measure of depressive symptomatology without the 5 somatic CES-D subscale items. ${ }^{18}$ Results of all models were unchanged by this modification of the outcome measure.

Finally, supplementary moderation analyses were undertaken to examine the possibility that the prediction of MDMA and meth/amphetamine use could vary by gender ${ }^{30}$ or pre-existing depressive symptoms - consistent with a stress-diathesis view in which vulnerable individuals would be more affected by drug use. ${ }^{31}$ Models 1 and 2 were replicated with interaction terms of MDMA use and meth/amphetamine use by gender, and by pre-existing depressive symptoms respectively. These terms were not statistically significant in any of the replicated models. 


\section{DISCUSSION}

MDMA and meth/amphetamine use in grade 10 predicted depressive symptoms one year later, independent of a number of important individual and contextual controls. This finding is consistent with animal and human studies suggesting long-term negative influences of synthetic drug use. ${ }^{8,9}$ Our results provide, to the best of our knowledge, the first compelling evidence that recreational MDMA and meth/amphetamine use places typically developing secondary school students at greater risk of experiencing depressive symptoms.

Compared to non-users, MDMA users and meth/amphetamine users in grade 10 were 1.7 and 1.6 times more likely to report depressive symptoms the following year, respectively. This association was not just an artefact of somatic symptoms found in the CES-D scale, which could reflect transient symptoms of drug use rather than longer-term affective outcomes. ${ }^{16}$ Contrary to earlier suggestions, ${ }^{30,31}$ this association did not vary by gender or antecedent depressive symptoms.

Further investigation indicated that concurrent meth/amphetamine and MDMA use (i.e. use of both substances, whether in the same drug-taking episode or not) accounted for most of the predictive power between the use of these drugs and subsequent depressive symptoms. This suggests additive or synergistic adverse effects of concurrent MDMA and meth/amphetamine use. Animal studies indicate that damage from MDMA and methamphetamine use can be synergistically enhanced by their concurrent use, especially within the same consumption episode. ${ }^{5}$ Many recreational users engage in simultaneous drug mixing and these users may be particularly at risk of developing negative affective consequences. ${ }^{5}$ Although some users may have developed a specific preference for one or the other, concurrent users might be more 
experienced or heavier users in contrast to MDMA or meth/amphetamine only users, who might be "recent initiators" or "experimenters." As such, our concurrent use variable could reflect adverse effects of both multiple drug use and of frequent and/or cumulative use. However, because this variable could primarily capture the intensive use of one substance more than the other and also because of the low prevalence of MDMA only and meth/amphetamine only use in our sample, it would be premature to discard the notion that MDMA or meth/amphetamine use has specific adverse effects.

The contribution of MDMA and meth/amphetamine use (in particular concurrent usage) to the prediction of subsequent depressive symptoms is relatively modest, but remarkable enough to be interpreted as nontrivial. Modest contributions can have significant clinical implications from a population-health perspective. This may be especially true in this case, given that drug-related consequences were considered in a low risk sample of youth at a time when the large majority of users were probably early in their trajectory of use. Depressive symptoms can influence delays or impediments in developmental milestones and social and occupational functioning. ${ }^{11}$ Any potential contribution of MDMA and meth/amphetamine use toward depressive symptoms should be taken seriously considering the risk of drug-induced neurotoxicity. Hence, from a cumulative risk perspective, adding the use of these drugs to the typical challenges and crises of adolescence might chart a life-course toward poorer mental health prognosis.

In addition to a prospective-longitudinal design, the main strength of this study was comprehensive control for important confounds that have not been consistently considered in previous work. A large number of individual and contextual characteristics antecedent to MDMA and meth/amphetamine use were included, particularly pre-existing depressive symptoms, and 
the use of other substances. Significant controls mainly reflected pre-existing psychological vulnerabilities (depressive symptoms, school anxiety) or individual difficulties (low conscientiousness, delinquency, and grade retention), reinforcing the need to control for such factors. ${ }^{7}$ Surprisingly, none of the substance use controls predicted subsequent depressive symptoms in our models, contradicting results from previous studies involving cannabis in particular. ${ }^{32}$ Nevertheless, findings have been mixed and typically depend on temporal and measurement factors. ${ }^{33}$

This study is not without limitations. First, despite our extensive control for other drug use, additive or interactive effects of meth/amphetamine and MDMA use coupled with other drugs cannot be ruled out. ${ }^{16}$ Second, all measures were self-reports, which is both a strength and limitation. The exact content of tablets sold as MDMA or meth/amphetamine eludes users, ${ }^{16}$ even if support exists for the validity of self-reported substance use. ${ }^{34}$ Third, attrition also poses a threat as in most longitudinal studies. Our confidence is reinforced by the fact that incomplete data were imputed and the direction and significance of results were largely similar with and without imputation.

Future research needs to better define which parameters of synthetic drug use matter most in predicting depressive outcomes in terms of frequency, quantity, trajectory and/or context of use. This includes specifying exactly how the concurrent use of these drugs increases the risk of such outcomes. Research that determines whether adolescent-onset versus adult-onset use has distinct associations with the development of depressive symptomatology, both at the neurobiological and functional levels, is also warranted. Longitudinal data, particularly mixed biological and selfreport, are most desirable to achieve this purpose. 
This study has important public health implications for adolescent populations. More evidence is still needed to secure more causal interpretations of meth/amphetamine and MDMA use in the development of depressive symptoms, and to determine the role of drug-induced 5-HT neurotoxicity. Nevertheless, our results concur with others and imply a 'principle of caution' in messages targeting adolescents, upholding that MDMA and meth/amphetamine use, particularly when concurrent, likely increases the risk of experiencing disruptions in affective symptomatology. 


\section{Acknowledgements.}

The authors wish to thank Ariane Descheneaux and Kim Archambault for their useful comments in the preparation of the manuscript. Everyone who contributed significantly is listed.

\section{Competing interests.}

None declared.

\section{Funding.}

This research was funded by a grant from the Fond Québécois de Recherche sur la Santé et la Société (FQRSC, 2007-NP-112947).

\section{Licence for Publication}

The Corresponding Author has the right to grant on behalf of all authors and does grant on behalf of all authors, an exclusive licence (or non exclusive for government employees) on a worldwide basis to the BMJ Publishing Group Ltd to permit this article (if accepted) to be published in JECH and any other BMJPGL products and sublicences such use and exploit all subsidiary rights, as set out in our licence (http://group.bmj.com/products/journals/instructions-for-authors/licence-forms). 


\section{REFERENCES}

1. Schwartz RH, Miller NS. MDMA (Ecstasy) and the Rave: A Review. Pediatrics. 1997;100(4):705-708.

2. Degenhardt L, Barker B, Topp L. Patterns of ecstasy use in Australia: Findings from a national household survey. Addiction. 2004;99(2):187-195.

3. Johnston LD, O’Malley PM, Bachman JG, et al. Monitoring the Future national survey results on drug use, 1975-2008: Volume I, Secondary school students (NIH Publication No. 09-7402). Bethesda, MD: National Institute on Drug Abuse; 2009.

4. Pederson W, Skrondal A. Ecstasy and new pattern of drug use: a normal population study. Addiction. 1999;94(11):1695-1706.

5. Clemens KJ, McGregor IS, Hunt GE, et al. MDMA, methamphetamine and their combination: possible lessons for party drug users from recent preclinical research. Drug Alcohol Rev. 2007;26(1):9-15.

6. Gouzoulis-Mayfrank E, Daumann J. Neurotoxicity of drugs of abuse- the case of methylenedioxyamphetamines (MDMA, ecstasy), and amphetamines. Dialogues Clin Neurosci. 2009;11(3):305-317.

7. Sumnall HR, Cole JC. Self-reported depressive symptomatology in community samples of polysubstance misusers who report Ecstasy use: a meta-analysis. $J$ Psychopharmacol. 2005;19(1):84-92.

8. Zweben JE, Cohen JB, Christian D, et al. Psychiatric symptoms in methamphetamine users. Am J Addict. 2004;13(2):181-90.

9. Parrott AC, Buchanan T, Scholey AB. Ecstasy/ MDMA attributed problems reported by novice, moderate and heavy recreational users. Hum Psychopharm Clin. 2002;17(6):309-312. 
10. Dahl RE. Adolescent brain development: a period of vulnerabilities and opportunities. Ann $N$ Y Acad Sci. 2004;1021:1-22.

11. Geller B, Zimerman B, Williams M, et al. Adult psychosocial outcome of prepubertal major depressive disorder. J Am Acad Child Adolesc Psychiatry. 2001;40(6):673677.

12. Degenhardt L, Coffey C, Moran P, et al. The predictors and consequences of adolescent amphetamine use: findings from the Victoria Adolescent Health Cohort Study. Addiction. 2007;102(7):1076-84.

13. Lieb R, Schuetz CG, Pfister H, et al. Mental disorders in ecstasy users: a prospectivelongitudinal investigation. Drug Alcohol Depend. 2002;68(2):195-207.

14. Yen CF, Chong MY. Comorbid psychiatric disorders, sex, and methamphetamine use in adolescents: a case-control study. Compr Psychiatry. 2006;47(3):215-20.

15. Sutcliffe CG, German D, Sirirojn B, et al. Patterns of methamphetamine use and symptoms of depression among young adults in northern Thailand. Drug Alcohol Depend. 2009;101(3):14651.

16. Cole JC, Bailey M, Sumnall HR, et al. The content of ecstasy tablets: implications for the study of their long-term effects. Addiction. 2002;97(12):1531-1536.

17. Radloff LS. The CES-D Scale: a self-report depression scale for research in the general population. Applied Psychological Measurement. 1977:1(3);385-401.

18. Radloff, LS. The use of the Center for Epidemiologic Studies Depression Scale in adolescents and young adults. $J$ Youth Adolesc. 1991;20(2):149-166.

19. Kessler RC, McGonagle KA, Zhao S, et al. Lifetime and 12-month prevalence of DSM-III-R psychiatric disorders in the United States: results from the National Comorbidity Survey. Arch Gen Psychiatry. 1994;51(1):8-19. 
20. Archambault I, Janosz M, Pagani L, et al. The multidimensionality of student engagement and its predictive relationship to high school dropout. J Adolesc. 2009;32(3):651-670.

21. Spence SH. Structure of anxiety symptoms among children: a confirmatory factor-analytic study. J Abnorm Psychol. 1997;106(2):280-297.

22. Le Blanc M, McDuff P, Fréchette M. Manuel sur des mesures de l'adaptation sociale et personnelle pour les adolescents québécois. Montréal: Université de Montréal, Groupe de recherche sur l'inadaptation psychosociale chez l'enfant; 1994.

23. Raven JC, Court JH, Raven, J. Standard Progressive Matrices. Oxford: Oxford Psychologists Press; 1996.

24. John OP. The "Big Five" factor taxonomy: Dimensions of personality in the natural language and in questionnaires. In: Pervin LA, ed. Handbook of Personality: Theory and Research. New York: Guilford press; 1990:66-100.

25. MacDonald KB. Effortful control, explicit processing, and the regulation of human evolved predispositions. Psych Rev. 2008;115(4):1012-1031.

26. Ganzeboom HBG, de Graff P, Treiman DA. Standard international socio-economic index of occupational status. Soc Sci Res. 1992;21(1):1-56.

27. Graham JW. Missing data analysis: making it work in the real world. Annu Rev Psychol. 2009;60:549-576.

28. Schafer JL. Analysis of Incomplete Multivariate Data. New York: Chapman \& Hall; 1997. 29. Mplus Users Guide [computer program]. Los Angeles, CA: Muthen LK and Muthen BO; 2006.

30. Reneman L, Booij J, de Bruin K, et al. Effects of dose, sex, and long-term abstention from use on toxic effects of MDMA (ecstasy) on brain serotonin neurons. Lancet. 2001;358:1864-69. 
31. Parrot AC. MDMA in humans: factors which affect the neuropsychobiological profiles of recreational ecstasy users, the integrative role of bioenergetic stress. J Psychopharmacol. 2006;20(2):147-163.

32. Hayatbakhsh MR, Najman JM, Jamrozik K, et al. Cannabis and anxiety and depression in young adults: a large prospective study. J Am Acad Child Adolesc Psychiatry. 2007;46(3):40817.

33. Rey JM, Sawyer MG, Raphael B, et al. Mental health of teenagers who use cannabis. Results of an Australian survey. Br J Psychiatry. 2002 Mar;180:216-21.

34. Barnea Z, Rahav G, Teichman M. The reliability and consistency of self-reports on substance use in a longitudinal study. Br J Addict. 1987;82(8):891-898 
Box 1.

What is already known on this subject?

- The use of the synthetic drugs MDMA (ecstasy) and meth/amphetamine (speed) tends to be associated with elevated depressive symptoms in adults.

- However, the existence and nature of this association in adolescents remains unclear.

What this study adds?

- This study provides evidence that the use of MDMA (ecstasy) and meth/amphetamine (speed) predicts subsequent depressive symptoms in adolescents beyond important individual and contextual confounders, including prior depressive symptoms and the use of other drugs.

- This suggests that synthetic drug use presents long-term risks for affective disturbance. 